\title{
Communication
}

\section{Ag-Yb Alloy-Novel Tunable Plasmonic Material}

\author{
Suetying Ching ${ }^{1}$, Chakming Chan ${ }^{1}$, Jack $\mathrm{Ng}^{2}$ and Kokwai Cheah ${ }^{1, *}$ \\ 1 Department of Physics and Institute of Advanced Materials, Hong Kong Baptist University, Kowloon Tong, \\ Hong Kong SAR, China; syching@hkbu.edu.hk (S.C.); 19482221@life.hkbu.edu.hk (C.C.) \\ 2 Department of Physics, Southern University of Science and Technology, Shenzhen 518055, China; \\ wuzh3@sustech.edu.cn \\ * Correspondence: kwcheah@hkbu.edu.hk
}

Citation: Ching, S.; Chan, C.; Ng, J.; Cheah, K. Ag-Yb Alloy-Novel

Tunable Plasmonic Material.

Photonics 2021, 8, 288. https:// doi.org/10.3390/photonics8070288

Received: 16 June 2021

Accepted: 14 July 2021

Published: 20 July 2021

Publisher's Note: MDPI stays neutral with regard to jurisdictional claims in published maps and institutional affiliations.

Copyright: (c) 2021 by the authors. Licensee MDPI, Basel, Switzerland. This article is an open access article distributed under the terms and conditions of the Creative Commons Attribution (CC BY) license (https:// creativecommons.org/licenses/by/ $4.0 /)$.

\begin{abstract}
Metals are commonly used in plasmonic devices because of their strong plasmonic property. However, such properties are not easily tuned. For applications such as spatial light modulators and beam steering, tunable plasmonic properties are essential, and neither metals nor other plasmonic materials possess truly tunable plasmonic properties. In this work, we show that the silver alloy silver-ytterbium $(\mathrm{Ag}-\mathrm{Yb})$ possesses tunable plasmonic properties; its plasmonic response strength can be adjusted as a function of $\mathrm{Yb}$ concentration. Such tunability can be explained in terms of the influence of $\mathrm{Yb}$ on bound charge and interaction of its dielectric with the dielectric of Ag. The change in transition characteristics progressively weakens Ag's plasmonic properties. With a spectral ellipsometric measurement, it was shown that the $\mathrm{Ag}-\mathrm{Yb}$ alloy thin film retains the properties of Ag with high transmission efficiency. The weakened surface plasmon coupling strength without dramatic change in the coupling wavelengths implies that the tunability of the Ag-Yb alloy is related to its volume ratio. The principle mechanism of the plasmonic change is theoretically explained using a model. This work points to a potential new type of tunable plasmonic material.
\end{abstract}

Keywords: plasmonics; metamaterials; subwavelength structures

\section{Introduction}

Plasmonics is a rapidly expanding research area in recent years with promising developments in both theoretical understanding and experimental techniques [1-6]. The plasmonic nanostructure has potential applications in super-resolution imaging [7], biosensing [8], wavefront engineering using metasurfaces [9-11], and plasmon-enhanced nonlinear optical processes [12-14]. Most plasmonic devices are made using noble metals because of their strong plasmonic property [15]. In particular, silver (Ag) and gold ( $\mathrm{Au}$ ) are considered good plasmonic materials, although they are intrinsically lossy in the visible and NIR regions; hence, transmissive plasmonic devices are not efficient, and alternate plasmonic material is needed for transmissive plasmonic devices. In developing alternate plasmonic materials [16-19], alloy of existing plasmonic materials seems to be a simple way to adjust the optical and/or electrical properties of the plasmonic nanostructure. There have been several reports on plasmonic properties of metal alloys and their fabrication [15,20-22]; alloying metals can alter the plasmonic characteristics of the metals and their plasmonic strength, and the energy of plasmonic resonance can be tuned [23]. However, most alloy metals remain reflective in the optical range. Only a few metal alloys exhibit transparency in the optical range; notable examples are $\mathrm{Ag}-\mathrm{Yb}$ and $\mathrm{Ag}-\mathrm{Mg}$. In an earlier work by the authors (S.Y.C.), it was shown that the former has better transparency than that of the latter [24]. In fact, Lee et al. took advantage of the improved transmission in the optical range of the Ag alloy to fabricate transparent, organic, light-emitting devices [25,26]. Although $\mathrm{Ag}-\mathrm{Yb}$ and $\mathrm{Ag}-\mathrm{Mg}$ alloys are being used as transparent electrodes, the physics behind the cause of transparency enhancement is not well understood. It is reasonable to assume that the underlying physics of transparency enhancement is the same for both Ag- 
$\mathrm{Yb}$ and $\mathrm{Ag}-\mathrm{Mg}$. Therefore, we focused on only one of them, namely, Ag-Yb, to elucidate the cause of transparency enhancement and the alloying effect on Ag's plasmonic properties.

In this work, we study the $\mathrm{Ag}$ alloy $\mathrm{Ag}-\mathrm{Yb}$, which was found to have a tunable plasmonic properties and transparency, which can be considered useful for a transmissive plasmonic device. The plasmonic characteristics of $\mathrm{Ag}-\mathrm{Yb}$ as a function of $\mathrm{Yb}$ concentration were investigated both theoretically and experimentally. As a demonstration of the alloy plasmonic properties, we utilized the near-field optical lithography technique to fabricate two-dimensional (2-D) disk arrays with a line width of $300 \mathrm{~nm}$ and a total area of $2.5 \mathrm{~mm}$ by $2.5 \mathrm{~mm}$. The disks were constituted of pure Ag and silver-ytterbium (Ag-Yb) alloy. In our previous study of a cavity superlens $[27,28]$, the near-field photolithography method was developed to fabricate nanostructures with a line width of around $150 \mathrm{~nm}$. We investigated the surface plasmon excitations of these $\mathrm{Ag}$ and $\mathrm{Ag}-\mathrm{Yb}$ disk arrays using a spectroscopic ellipsometer. It was shown that the surface plasmon excitation was diluted in the $\mathrm{Ag}-\mathrm{Yb}$ alloy device when compared to the control device (pure Ag disk array). Our theoretical model shows that the excitation dilution is due to the dilution of the free charges after introducing the $\mathrm{Yb}$. This $\mathrm{Ag}-\mathrm{Yb}$ alloy opens an important avenue for transmissive and tunable plasmonic devices.

\section{Fabrication}

Thin film samples of the alloys were prepared on plain glass and silicon (Si) wafer. Films of 20-120 nm thickness were prepared using thermal evaporation. For the Ag- $\mathrm{Yb}$ film, thermal co-deposition of the two materials was used. The volume ratio of the mixture of the materials was controlled by tuning the deposition rates of individual sources such that the desired mixed ratio was obtained. The ratio of the $\mathrm{Ag}: \mathrm{Yb} 20 \mathrm{~nm}$ thick film was $7: 3$, and it was deposited with deposition rate of $1.4 \AA / \mathrm{s}$ and $0.6 \AA / \mathrm{s}$ for the $\mathrm{Ag}$ and $\mathrm{Yb}$, respectively.

For the two dimensional disk array plasmonic nanostructures, near-field photolithography was used. Figure 1a shows the schematic of the disk array. A layer of $50 \mathrm{~nm} \mathrm{Cr}$ film was deposited on a quartz substrate via the radio frequency magnetron sputtering technique followed by a layer of $400 \mathrm{~nm}$ e-beam resist (ZEP520A) spin-coated on the as-deposited $\mathrm{Cr}$ layer. The e-beam resist was patterned with squared holes with a size of 300 by $300 \mathrm{~nm}$, and the period was $600 \mathrm{~nm}$; the spacing was sufficiently large so that the nanostructure could be considered as standalone. Using near-field photolithography, the hole array pattern on the $\mathrm{Cr}$ mask was transferred to a layer of $250 \mathrm{~nm}$ photoresist (Hir1075) spin-coated on glass substrates with a thickness of $0.7 \mathrm{~mm}$. Two samples were prepared: one of the patterned glass substrates with $40 \mathrm{~nm}$ Ag deposited and a substrate where $\mathrm{Ag}-\mathrm{Yb}$ film was deposited, using thermal evaporation for both. The concentration of $\mathrm{Yb}$ in $\mathrm{Ag}$ was $30 \mathrm{wt}$. \%. After the removal of the photoresist via the metal lift-off process, 2D disk arrays were obtained. The disk arrays of $\mathrm{Ag}$ and $\mathrm{Ag}-\mathrm{Yb}$ were confirmed by SEM (Figure $1 \mathrm{~b}, \mathrm{c}$ ) with each round disk carrying a diameter of $300 \mathrm{~nm}$. The spacing between each nanostructure was sufficiently large $(600 \mathrm{~nm})$ such that there was no interaction between them. Therefore, the spectroscopy results obtained are a collective response of the individual nanostructure. Photographs of the $2.5 \mathrm{~mm}$ by $2.5 \mathrm{~mm}$ squared sample areas of the nanostructures are shown in Figure 1d,e.

XRD (X-ray diffraction) was performed on samples of $\mathrm{Ag}$ and $\mathrm{Ag}-\mathrm{Yb}$ alloy with different percentage of $\mathrm{Yb}$ (Figure 2a). The XRD results show that Ag crystallites becoming progressively smaller with increasing $\mathrm{Yb}$ concentration, and, therefore, no $\mathrm{Yb}$ crystallization was observed. From Figure 2a, we derived the crystallite size using the Scherrer equation $[29,30]$ :

$$
L=\frac{K \lambda}{B \cos \theta}
$$

where $K$ is the Scherrer constant, which is dimensionless; $\lambda$ is the wavelength of the incident radiation; $B$ is the line breadth of the peak profile in radians; and $\theta$ is the diffraction angle of reflection. The Scherrer constant $K$ depends on peak shape, crystallite shape, and size 
distribution. The constant $K$ varies from 0.89 for spherical geometry to 0.94 for cubic geometry. It is assumed that the metal alloy has a uniformly distributed spherical crystals in cubic symmetry, so $K=0.94$ is used. Figure $2 b$ shows that the size of the metal alloy crystallite varies as a function of $\mathrm{Yb}$ concentration; when $\mathrm{Yb}$ concentration in the metal alloy is more than $20 \%$, the crystallite size is $\leq 5 \mathrm{~nm}$; with $50 \% \mathrm{Yb}$, the crystallite size is only $2.3 \mathrm{~nm}$. Thus, the metal alloy becomes amorphous. Furthermore, the XRD peaks observed in the $\mathrm{Ag}-\mathrm{Yb}$ alloy are those of $\mathrm{Ag}$; i.e., $\mathrm{Yb}$ did not form any crystallite, and, in fact, the alloy film became amorphous with the increase in $\mathrm{Yb}$ concentration.

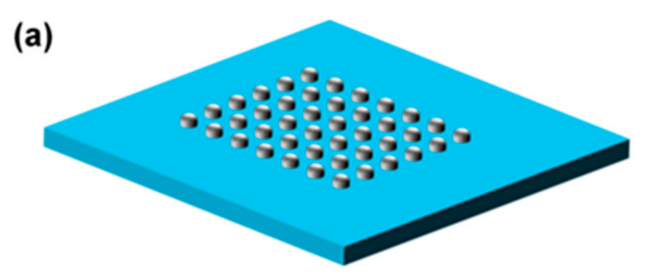

(b)

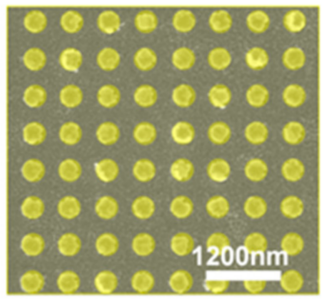

(d)

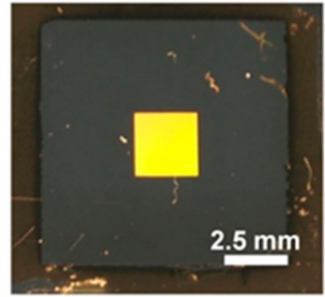

(c)

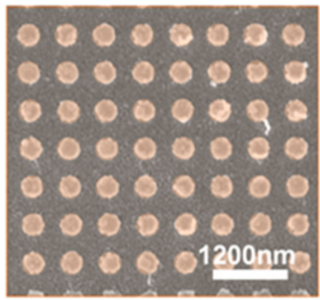

(e)

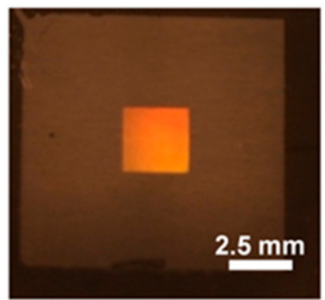

Figure 1. (a) Illustration of a disk array (not-to-scale); (b,c) SEM (Scanning Electron Microscope) images of the 2-D Ag (b) and $\mathrm{Ag}-\mathrm{Yb}(\mathbf{c})$ disk arrays where the diameter of each disk is about $300 \mathrm{~nm}$; $(\mathbf{d}, \mathbf{e})$ photographs of the $2 \mathrm{D}$ hole array $\mathrm{Ag}(\mathbf{d})$ and $\mathrm{Ag}-\mathrm{Yb}(\mathbf{e})$ nanostructures on glass substrates with a pattern size of $2.5 \mathrm{~mm} \times 2.5 \mathrm{~mm}$.

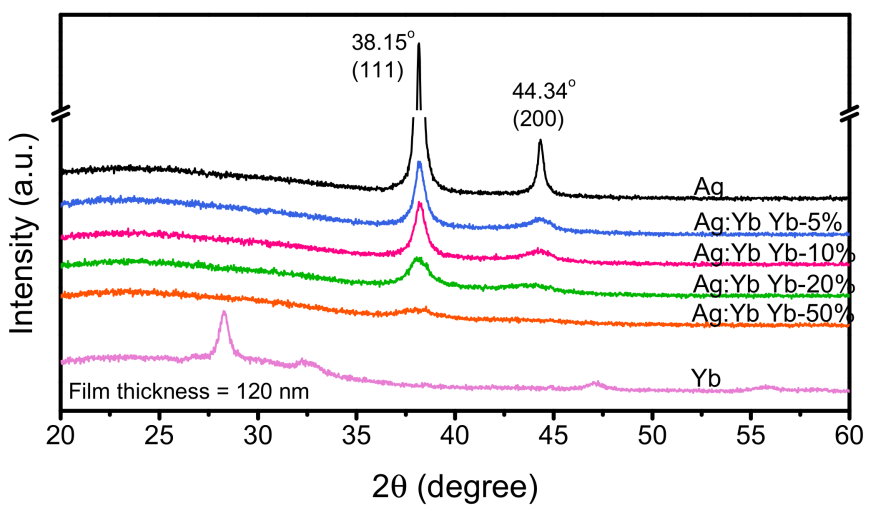

(a)

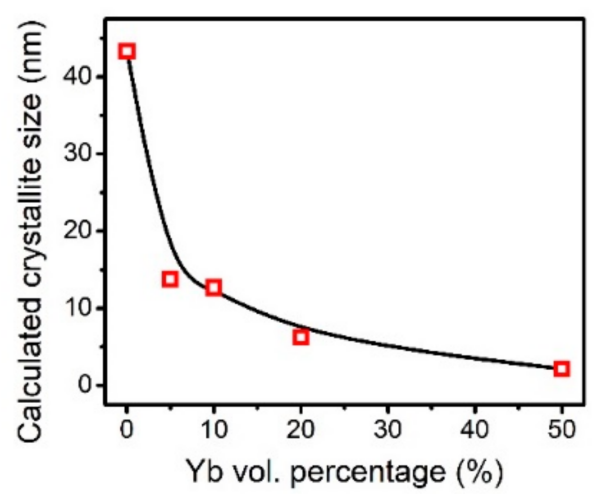

(b)

Figure 2. (a) XRD of Ag, $\mathrm{Yb}$, and Ag-Yb thin films. For Ag- $\mathrm{Yb}$ alloy film, the $\mathrm{Yb}$ concentration is 5\%, 10\%, 20\%, and 50\%. Only Ag crystallite peaks were observed in the Ag-Yb alloy, the XRD peak(s) is very weak at 50\% Yb. No Yb XRD peaks were observed for the Ag-Yb alloy films. (b) Using the Sherrer equation [30,31], the Ag crystallite sizes were derived. The largest crystallite's size is $43 \mathrm{~nm}$ at $0 \% \mathrm{Yb}$, and the smallest crystallite size is $2.3 \mathrm{~nm}$ at $50 \% \mathrm{Yb}$. 


\section{Results and Discussion}

For $\mathrm{Ag}-\mathrm{Yb}$ film, the transmission increased across the visible range. However, the $\mathrm{Ag}$ resonance at $320 \mathrm{~nm}$ and the $\mathrm{Ag}-\mathrm{Yb}$ film weakened the resonant peak that blue-shifted to $305 \mathrm{~nm}$ (Figure 3). In fact, the Ag resonant wavelength blue-shifted as $\mathrm{Yb}$ concentration increased. The transmission of $\mathrm{Ag}-\mathrm{Yb}$ improved on average by $10 \%$ across the visible range in comparison to that of $\mathrm{Ag}$ film, and the transmission over the visible range increased with the increase in $\mathrm{Yb}$ concentration. However, the electrical conductivity decreased with the increase in $\mathrm{Yb}$ concentration. In this work, a 7:3 ratio of $\mathrm{Ag}: \mathrm{Yb}$ was chosen, as this allowed for an acceptable level of electrical conductivity for potential applications, such as in transparent conducting electrodes, as well as transparency for transmission applications and investigations.

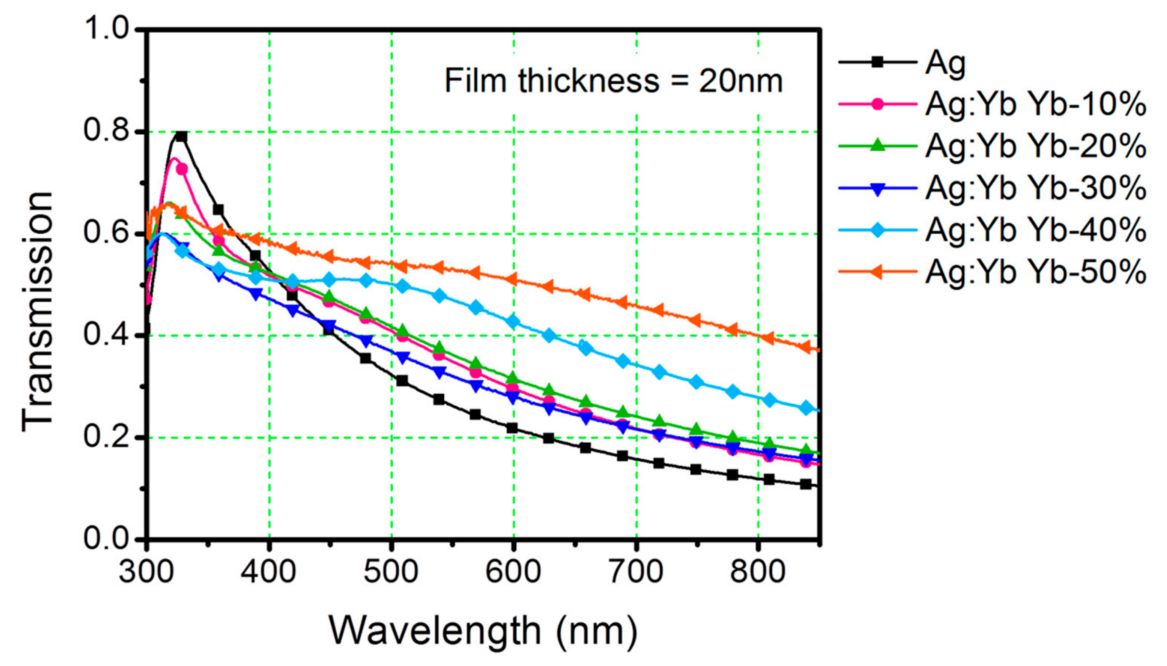

Figure 3. Transmission of $\mathrm{Ag}$ and $\mathrm{Ag}-\mathrm{Yb}$ films; typically, the $\mathrm{Ag}$ film has resonant transmission at $320 \mathrm{~nm}$ and the transmission drops progressively across the visible range. In contrast, the Ag- $\mathrm{Yb}$ film has a weakened resonant at a wavelength of $305 \mathrm{~nm}$, and transmission improves on average by $10 \%$ across the visible range.

Figure 3 shows that there is was continuous enhancement in the transmission in the visible and NIR regions with an increasing concentration of $\mathrm{Yb}$ in Ag by up to around 50 wt. \%. The electrical conductivity, however, decreased with the increase in $\mathrm{Yb}$ concentration in $\mathrm{Ag}-\mathrm{Yb}$. There was a small blue shift in the plasmonic resonant peak in $\mathrm{UV}$ at $14 \mathrm{~nm}$ with the increase in the doping concentration of $\mathrm{Yb}$. The transmission of Ag dropped below $40 \%$ at the optical wavelength longer than $450 \mathrm{~nm}$. However, when $\mathrm{Yb}$ was doped into $\mathrm{Ag}$ with concentration from 10 to $50 \%$, the transmission from 450 to $800 \mathrm{~nm}$ increased; at $450 \mathrm{~nm}$, the transmission increased from 40 to $55 \%$, and at $800 \mathrm{~nm}$, the transmission increased from 12 to $63 \%$. As the transmission in the long wavelength increased, the transmission in the blue/UV (300-400 $\mathrm{nm}$ ) region dropped; transmission at the plasmonic resonant peak dropped to only $60 \%$ at a $\mathrm{Yb}$ doping concentration of $40 \%$. When the $\mathrm{Yb}$ doping concentration was $50 \%$ or above, the transmission began to resemble that of $\mathrm{Yb}$. Thus, the transmission of the Ag-Yb alloy in the optical range could be tuned by introducing different $\mathrm{Yb}$ concentrations into Ag. The electrical conductivity of the $\mathrm{Ag}-\mathrm{Yb}$ also varied with $\mathrm{Yb}$ concentration. The sheet resistance of the pure Ag film was $1.7 \Omega / \square$, whereas for $A g-Y b$, the sheet resistance increased with the increase in $\mathrm{Yb}$ concentration; at 30 wt. \% of $\mathrm{Yb}$, it was $18.1 \Omega / \square$. Figure 4 depicts the change in electrical conductivity as a function of $\mathrm{Yb}$ concentration. It shows that the electrical conductivity dropped exponentially between 0 and $40 \% \mathrm{Yb}$; when $\mathrm{Yb}$ concentration was higher than $40 \%$, the electrical conductivity reached a stable value at $0.08 \times 10^{5} \mathrm{~S} / \mathrm{cm}$. The Ag-Yb alloy with a $\mathrm{Yb}$ concentration of 50 and $30 \%$ was used as a transparent electrode for OLED [24,26]. A higher $\mathrm{Yb}$ concentration percentage has higher optical transmission (Figure 3); however, 
the sheet resistance is two orders of magnitude higher. Therefore, this must be taken into consideration when developing OLED devices.

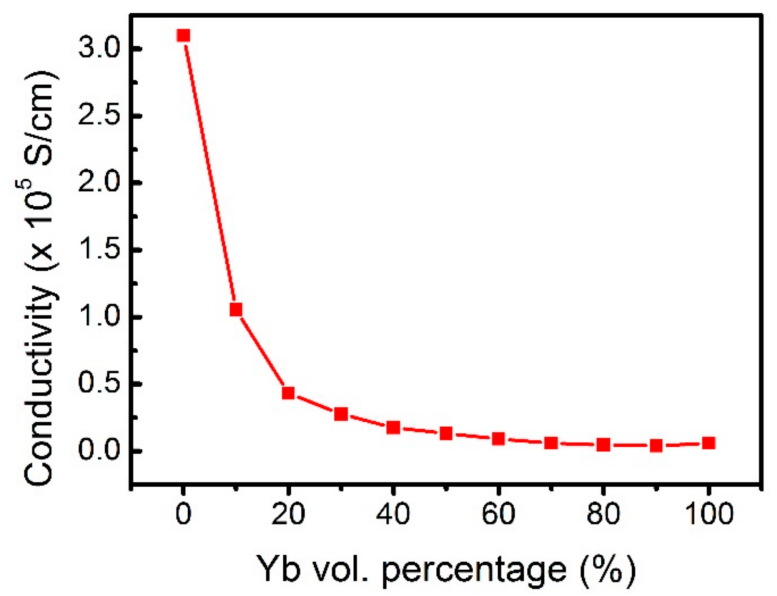

Figure 4. Electrical conductivity of Ag- $\mathrm{Yb}$ alloy film as a function of $\mathrm{Yb}$ concentration; the presence of $\mathrm{Yb}$ in the alloy exponentially reduces the conductivity, reaching a stable value of $0.08 \times 10^{5} \mathrm{~S} / \mathrm{cm}$ at a $\mathrm{Yb}$ concentration $\geq 40 \%$, with a drop of $400 \times$ in conductivity.

The surface plasmon polariton (SPP) excitation of the pure Ag and $\mathrm{Ag}-\mathrm{Yb}$ plasmonic nanostructures was determined by angle-resolved ellipsometric spectra (Figure 5a,b). Tan $\Psi$ is the absolute value of the $p$ and $s$ polarization reflectance amplitude ratio between $r_{\mathrm{p}}$ and $r_{\mathrm{s}}$, and only $p$-polarized light excites the SPP. When there is a plasmonic coupling of the incident light with the nanostructure, the reflection intensity of $p$-polarized light suddenly decreases, while that of s-polarized light remains unchanged. Hence, the SPP excitations are depicted by the dips in Figure 5, which are situated at the infrared (IR) spectral region for both samples. The spacing between each nanostructure is $600 \mathrm{~nm}$; i.e., there is no plasmonic interaction between them. Thus, the spectra in Figure 5 represent the collective response from an individual nanostructure.

(a)

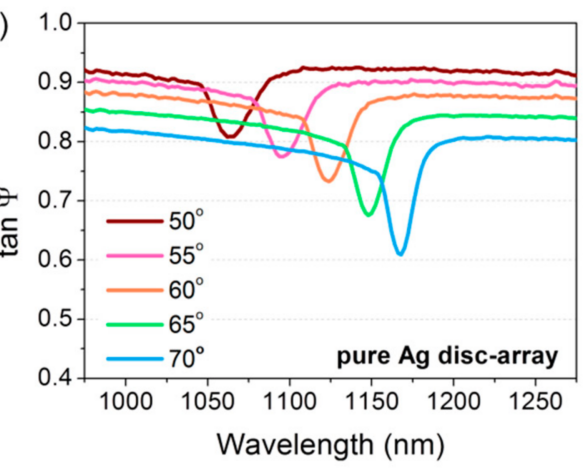

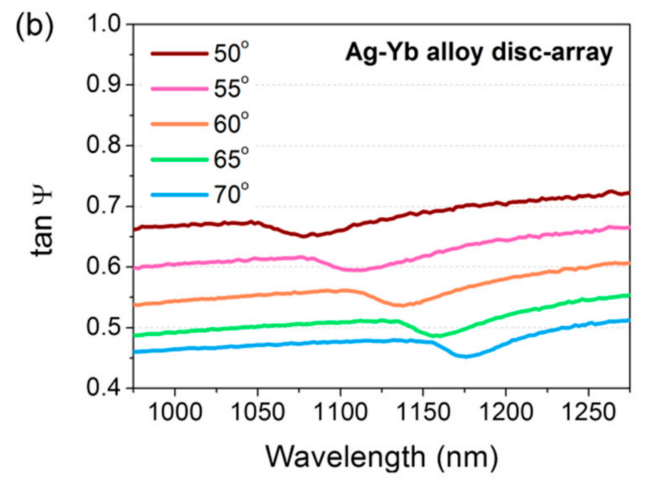

Figure 5. Amplitude ratios of the ellipsometric measurements at incident angles from $50^{\circ}$ to $70^{\circ}$ for pure $\mathrm{Ag}(\mathbf{a})$ and $\mathrm{Ag}-\mathrm{Yb}(\mathbf{b})$ alloy disk array devices.

From Figure 5, we can derive the dispersion of the resonant (dip) wavelength as a function of the incident angle. Figure 6 compares the experimental and simulated resonant wavelengths as a function of incident angles. The simulated resonant wavelengths agreed well with the measured values, with an error of $\pm 0.4 \%$ after adjusting the period $p$ to $605 \mathrm{~nm}$ for the $\mathrm{Ag}-\mathrm{Yb}$ disk array. We attribute this $5 \mathrm{~nm}$ variance to systematic variation in sample fabrication, and it could cause $\sim 10 \mathrm{~nm}$ offset of the coupling wavelengths for the alloy device. The result shows that the $\mathrm{Ag}-\mathrm{Yb}$ alloy plasmonic resonant characteristic still 
resembles that of Ag. Thus, it is the free electrons from the Ag that contribute most to the plasmonic characteristics.

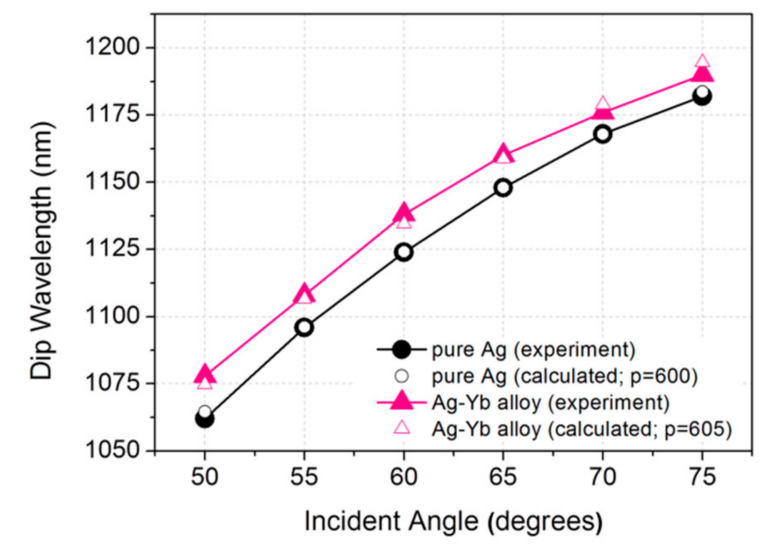

Figure 6. SPP coupling wavelengths acquired from the amplitude ratios of the ellipsometric measurements at varied incident angles of pure $\mathrm{Ag}$ and $\mathrm{Ag}-\mathrm{Yb}$ disk arrays and theoretical calculations.

From the ellipsometry measurement, the dielectric of $\mathrm{Ag}-\mathrm{Yb}$ alloy was also derived (Figure 7). The shift in the real part of the dielectric, $\varepsilon_{1}$, confirms that the plasmonic resonant frequency blue-shifted with the increase in $\mathrm{Yb}$ concentration. Moreover, the imaginary part of the dielectric, $\varepsilon_{2}$, i.e., the transition point, remained unchanged; however, it broadened with the increase in $\mathrm{Yb}$ concentration; that is, the Ag dielectric characteristics were diluted by $\mathrm{Yb}$. Therefore, although the $\mathrm{Ag}-\mathrm{Yb}$ alloy essentially retained the $\mathrm{Ag}$ plasmonic characteristics, its plasmonic resonant intensity decreased with the increase in $\mathrm{Yb}$ concentration, and the transmission across the optical range increased. This implies that when $\mathrm{Yb}$ is introduced into $\mathrm{Ag}$, there is a reduction in free electron density. In order to confirm this hypothesis, a simulation was performed.

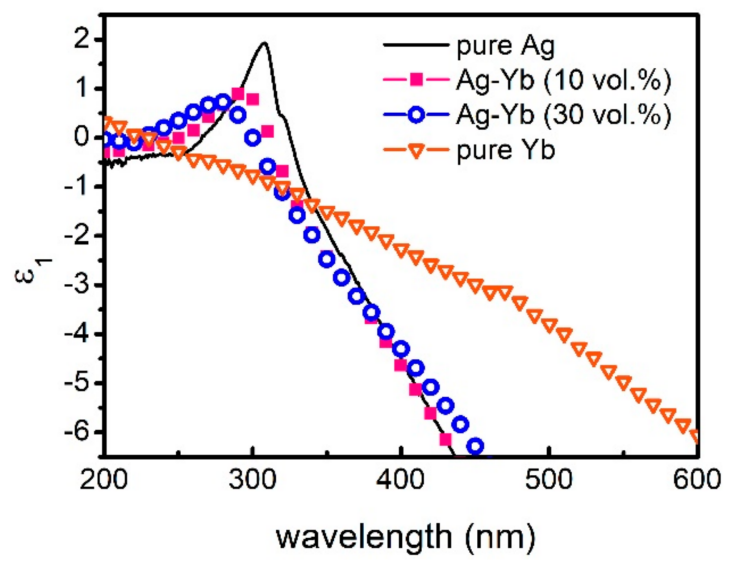

(a)

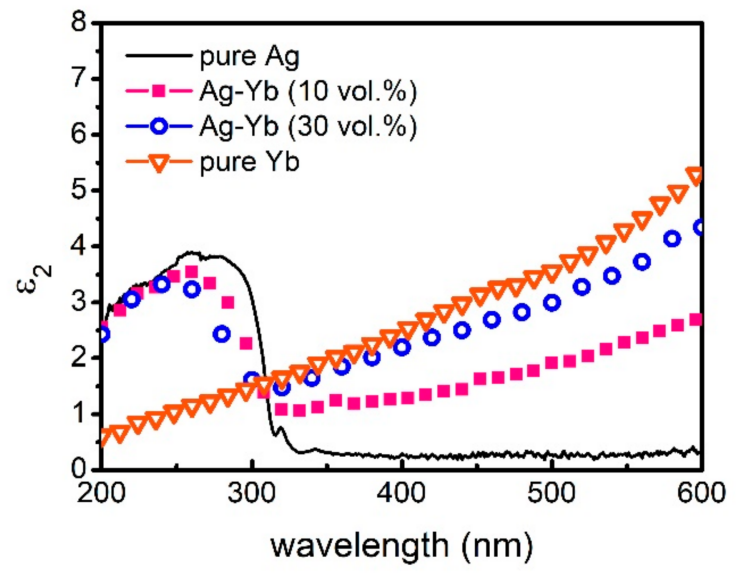

(b)

Figure 7. Change in dielectric of $\mathrm{Ag}-\mathrm{Yb}$ alloy as a function of $\mathrm{Yb}$ concentration. (a) $\varepsilon_{1}$ resonant peak shifts towards higher photon energy with increasing $\mathrm{Yb}$ concentration. (b) $\varepsilon_{2}$ transition point remains fixed, but the transition gradient drops with increasing $\mathrm{Yb}$ concentration.

The simulation is based on the momentum matching condition of SPP on one-dimensional (1D) grating and can be described by following equations [2]:

$$
k_{s p}(\omega)=k_{0}(\omega) \sin (\theta)+m G_{0}
$$




$$
\therefore k_{s p}(\omega)=k_{0}(\omega) \sqrt{\varepsilon_{\text {metal }} /\left(\varepsilon_{\text {metal }}+1\right)}
$$

where $k_{s p}$ is the wave vector of the surface plasmon excited at metal/air interface, $k_{0}$ is the vacuum wave vector of incident light, $\omega$ is the frequency, $\theta$ is the angle of incidence, $m$ is a non-zero integer indicating the order of surface plasmon coupling, $G_{0}$ is the reciprocal lattice vector $\left(G_{0}=2 \pi / p\right.$, where $p$ is the period), and $\varepsilon_{\text {metal }}$ is the permittivity of the metal. In this work, $m=-1$ was chosen to excite backward-propagating SPs, and $\varepsilon_{\text {metal }}$ is the permittivities of pure $\mathrm{Ag}$ or $\mathrm{Ag}-\mathrm{Yb}$.

Modelling of the $\mathrm{Ag}-\mathrm{Yb}$ alloy thin film was carried out by using finite element simulation (Comsol Multiphysics) [31] to elucidate the plasmonic characteristic change due to the mixing of $\mathrm{Yb}$ into Ag. In the simulation, the unit cell was composed of composite material of $\mathrm{Ag}$ and $\mathrm{Yb}$ (Figure 8), where the periodic boundary condition was applied along $\mathrm{x}$, and the incident plane wave was illuminated from the top. The size of the Ag and $\mathrm{Yb}$ block was chosen such that the entire system could be described by effective medium theory. We are aware that the structure shown in Figure 8 may not fully represent the real structure, whose mixing can be more complicated. Nevertheless, irrespective of the geometry of mixing, the size of the unit cell, and the effective medium model used, these models should all give qualitatively correct results, as shown in this work. In fact, the dielectric constant of thin film is known to be different from the bulk. Thus, the use of the bulk dielectric constant of bulk silver and ytterbium in the simulation of the thin film is at best a qualitative approach, and, in our case, a good qualitative approach.

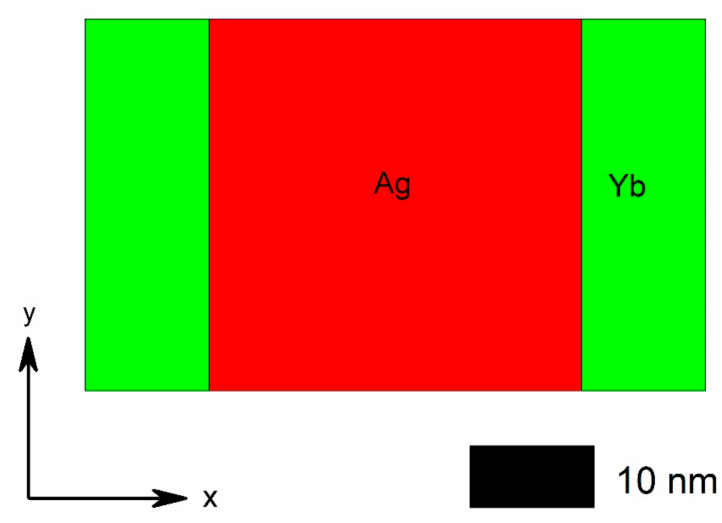

(a)

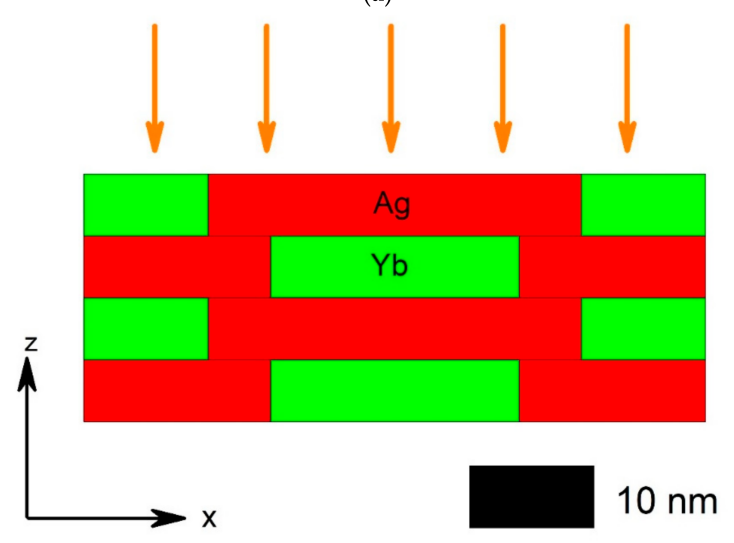

(b)

Figure 8. Composite of $\mathrm{Ag}$ and $\mathrm{Yb}$ in a unit cell used in the simulation (finite element simulation using Comsol Multiphysics). (a) Top view of the unit cell. (b) Side view of the unit cell showing the four-layer composite. The $20 \mathrm{~nm}$ alloy film was divided into four layers. Then, the composite resembled a $2 \mathrm{D}$ structure, allowing a more realistic simulation. Periodic boundary conditions were applied in the $\mathrm{x}$ direction. 
The $20 \mathrm{~nm}$ alloy film was divided into numerous multi-layer 2D structures to mimic an alloy. The simulation (Figure 9) agrees well with the experimental result (Figure 3); both the plasma peak that shifts monotonically towards the shorter wavelength and the increase in transmittance on the long wavelength side agree with the experimental results. However, the amount of shift calculated is less than that of the experiment. This shows that mixing is even more thorough than one can expect from effective medium theory, making our approach promising.

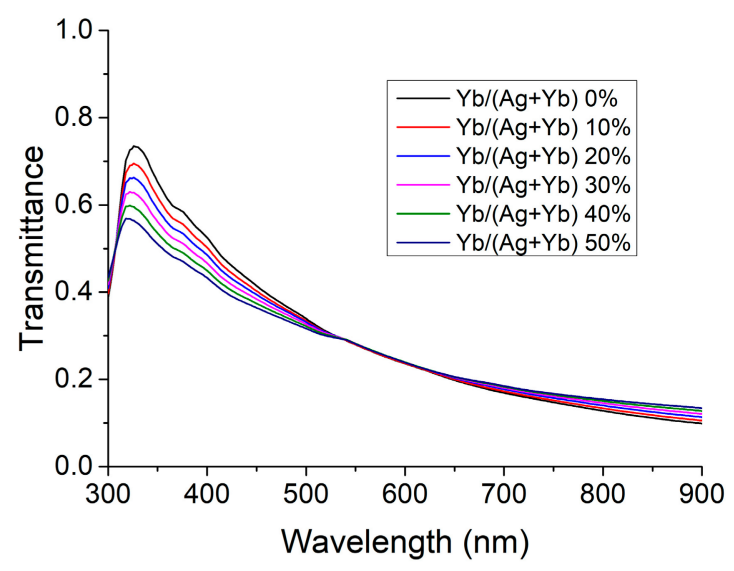

(a)

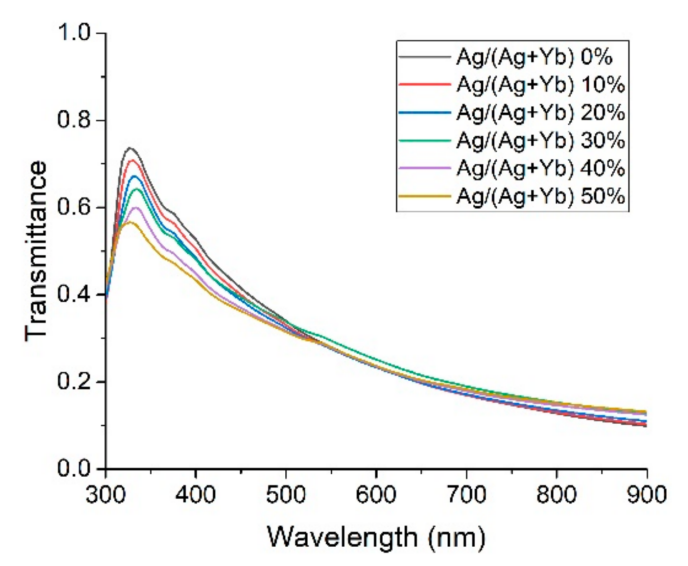

(b)

Figure 9. The calculated transmittance for the unit cell. (a) y-polarized incident light. (b) x-polarized incident light.

From the Drude model, the plasma frequency of a metal is given by

$$
\omega_{p}^{2}=n e^{2} / m \varepsilon_{0}
$$

where $n$ is the electron density, $e$ is the electron charge, $m$ is the electron mass, and $\varepsilon_{0}$ is the fee space dielectric.

In our composite model, the unit cell is subwavelength, so the electron density $n$ should be that of the unit cell average. This gives

$$
n=(1-X) n_{A g}+X n_{Y b}
$$

where $\mathrm{X}$ is the percentage of $\mathrm{Yb}$ in the alloy by volume, and $n_{A g}$ and $n_{Y b}$ are the electron densities of $\mathrm{Ag}$ and $\mathrm{Yb}$, respectively.

Since the electron density of $\mathrm{Yb}$ is higher than that of the Ag, the electron density increases with $\mathrm{Yb}$ concentration, and, therefore, the peak associated with plasma frequency shifts toward a higher frequency or shorter wavelength.

Figure 9a agrees with the experimental measurement; first, the plasma peak is monotonically shifting towards the shorter wavelength. Second, the transmittance on the long wavelength side is increased. For Figure $9 b$, with the increase $\mathrm{Yb}$ concentration, the plasmonic peak shifts about the Ag resonant peak and, therefore, disagrees with the experimentally observed blue shift. This is because for the x-polarization, the electrons are driven to move across the $\mathrm{Ag}$ and $\mathrm{Yb}$ boundary. This results in additional boundary scattering, which should not be present in a real homogeneous alloy.

To further theoretically explain the experimental observed change in the Ag plasmonic property, the Drude model is used. The dielectric constant of metal is approximately given by

$$
\varepsilon_{\text {metal }}=\varepsilon_{b}-\omega_{p}^{2} / \omega^{2}
$$


Here, $\varepsilon_{b}$ is the contribution from the bound electrons, which is not equal to 1 in general. For $\mathrm{Ag}$ and $\mathrm{Yb}$, the dielectric constants are

$$
\begin{gathered}
\varepsilon_{A g}=\varepsilon_{A g}^{b}-\omega_{A g}^{2} / \omega^{2}, \\
\varepsilon_{Y b}=\varepsilon_{Y b}^{b}-\omega_{Y b}^{2} / \omega^{2}
\end{gathered}
$$

Here, $\varepsilon_{Y b}^{b}<<\varepsilon_{A g}^{b}$ and $\omega_{Y b}^{2}<\omega_{A g}^{2}$, which can be inferred from the measured dielectric constants for $\mathrm{Ag}$ and $\mathrm{Yb}$. For the mixture with $\mathrm{Yb}$ concentration $\mathrm{X}$, the dielectric constant in a first-order approximation is given by the volume average:

$$
\varepsilon_{m i x}=(1-X) \varepsilon_{A g}+X \varepsilon_{Y b}
$$

Solving for the plasma frequency defined by $\varepsilon_{m i x}=0$, one has

$$
\left(\frac{\lambda}{2 \pi c}\right)^{2}=\frac{\varepsilon_{A g}^{b}}{\omega_{A g}^{2}}\left(\frac{1-X\left[1-\varepsilon_{Y b}^{b} / \varepsilon_{A g}^{b}\right]}{1-X\left[1-\omega_{Y b}^{2} / \omega_{A g}^{2}\right]}\right) \approx \frac{\varepsilon_{A g}^{b}}{\omega_{A g}^{2}}\left(\frac{1-X}{1-X\left[1-\omega_{Y b}^{2} / \omega_{A g}^{2}\right]}\right)
$$

where the right most term follows from $\varepsilon_{Y b}^{b}<<\varepsilon_{A g}^{b}$. Given that the quantity in the angular bracket is less than 1, Equation (9) is a decreasing function of concentration $X$. This explains why the peak wavelength decreases with concentration. The blue shift is caused by $\varepsilon_{Y b}^{b}<<\varepsilon_{A g}^{b}$ i.e., the bound charges of silver have significantly stronger contributions than those of ytterbium. This explains the small blue shift observed for the resonant wavelength, as shown in Figure 3. This shows that the plasmonic characteristic of Ag- $\mathrm{Yb}$ film is similar to that of pure Ag film, and the main difference is the increasingly transparency of the alloy film at the long wavelength side.

For the long wavelength side, one has

$$
\begin{aligned}
\varepsilon_{\text {mix }}= & (1-X)\left(\varepsilon_{A g}^{b}-\omega_{A g}^{2} / \omega^{2}\right)+X\left(\varepsilon_{Y b}^{b}-\omega_{Y b}^{2} / \omega^{2}\right) \\
\approx & (1-X)\left(-\omega_{A g}^{2} / \omega^{2}\right)+X\left(-\omega_{Y b}^{2} / \omega^{2}\right) \\
= & -\omega_{A g}^{2} / \omega^{2}+X\left(\omega_{A g}^{2}-\omega_{Y b}^{2}\right) / \omega^{2}
\end{aligned}
$$

In Equation (10), the second term in the third line is positive, as $\omega_{Y b}^{2}<\omega_{A g}^{2}$. Thus, the resultant dielectric constant is negative. Furthermore, the first term in Equation (10) is large and negative, but as $X$ increases, it becomes less negative. Since a highly negative dielectric constant represents strong reflection, the material becomes more transparent with increasing $\mathrm{Yb}$ concentration. This agrees well with the experimental result (Figure 2).

In summary, the condition $\varepsilon_{Y b}^{b}<<\varepsilon_{A g}^{b}$, which is solely related to the bound charge, provides a blue-shifting peak in the short wavelength side, while the condition $\omega_{Y b}^{2}<\omega_{A g}^{2}$ which is solely related to the free charge, provides increasing transparency at the long wavelength side. If loss is included in the analytical model, the simulated result should agree with the experimental result. However, the model would be too complex for elucidation of the underlining physics.

\section{Conclusions}

The plasmonic property of $\mathrm{Yb}$-doped Ag was experimentally and theoretically investigated. A gradual decrease in the resonant plasmonic frequency with a corresponding blue shift in the resonant peak wavelength was observed with the increase in $\mathrm{Yb}$ doping concentration. There was an increase in optical transparency at the longer wavelength region of the optical range with the increase in $\mathrm{Yb}$ concentration. The observed phenomena was explained theoretically: the bound charges, i.e., the trapped electrons, are responsible for the resonant peak blue shift, which is a consequence of $\varepsilon_{Y b}^{b}<<\varepsilon_{A g}^{b}$, whereas the lower plasmonic resonant frequency of the dopant, $\mathrm{Yb}$, gives rise to greater transmission 
at the longer wavelength region. Therefore, this work provides a mechanism that allows metal alloys to have a tunable plasmonic property with an increase in optical transparency. This opens up the possibility of the introduction of a new class of plasmonic 'transparent' metal alloys.

Author Contributions: The experiments were performed by S.C.; simulation was performed by C.C. and J.N. K.C. analyzed the result and wrote the paper with support from J.N. All authors have read and agreed to the published version of the manuscript.

Funding: The research was funded by the Hong Kong Research Grant Council, AoE/P-02/12 and 12303019 .

Institutional Review Board Statement: Not applicable.

Informed Consent Statement: Not applicable.

Acknowledgments: The authors acknowledge the financial support from the Hong Kong Research Grant Council, AoE/P-02/12 and 12303019.

Conflicts of Interest: The authors declare no conflict of interest.

\section{References}

1. Raether, H. Surface Plasmons on Smooth and Rough Surfaces and on Gratings; Springer: Berlin/Heidelberg, Germany, 1988.

2. Ebbesen, T.W.; Lezec, H.J.; Ghaemi, H.F;; Thio, T.; Wolff, P.A. Extraordinary optical transmission through sub-wavelength hole arrays. Nat. Cell Biol. 1998, 391, 667-669. [CrossRef]

3. Barnes, W.L.; Dereux, A.; Ebbesen, T.W. Surface plasmon subwavelength optics. Nature 2003, 424, 824-830. [CrossRef] [PubMed]

4. Maier, S.A. Plasmonics: Fundamentals and Applications; Springer Science \& Business Media: New York, NY, USA, 2007; pp. 1-9.

5. de Abajo, F.J.G. Colloquium: Light scattering by particle and hole arrays. Rev. Mod. Phys. 2007, 79, 1267-1290. [CrossRef]

6. Garcia-Vidal, F.; Martín-Moreno, L.; Ebbesen, T.W.; Kuipers, L. Light passing through subwavelength apertures. Rev. Mod. Phys. 2010, 82, 729-787. [CrossRef]

7. Lu, D.; Liu, Z. Hyperlenses and metalenses for far-field super-resolution imaging. Nat. Commun. 2012, 3, 1205. [CrossRef]

8. Sönnichsen, C.; Reinhard, B.M.; Liphardt, J.; Alivisatos, P. A molecular ruler based on plasmon coupling of single gold and silver nanoparticles. Nat. Biotechnol. 2005, 23, 741-745. [CrossRef]

9. Yu, N.; Genevet, P.; Kats, M.; Aieta, F.; Tetienne, J.-P.; Capasso, F.; Gaburro, Z. Light Propagation with Phase Discontinuities: Generalized Laws of Reflection and Refraction. Science 2011, 334, 333-337. [CrossRef]

10. Ni, X.; Emani, N.K.; Kildishev, A.; Boltasseva, A.; Shalaev, V.M. Broadband Light Bending with Plasmonic Nanoantennas. Science 2011, 335, 427. [CrossRef]

11. Chen, X.; Huang, L.; Mühlenbernd, H.; Li, G.; Bai, B.; Tan, Q.; Jin, G.; Qiu, C.-W.; Zhang, S.; Zentgraf, T. Dual-polarity plasmonic metalens for visible light. Nat. Commun. 2012, 3, 1198. [CrossRef]

12. Kauranen, M.; Zayats, A.V. Nonlinear plasmonics. Nat. Photonics 2012, 6, 737-748. [CrossRef]

13. Liu, H.; Li, G.; Li, K.F.; Chen, S.M.; Zhu, S.N.; Chan, C.T.; Cheah, K.W. Linear and nonlinear Fano resonance on two-dimensional magnetic metamaterials. Phys. Rev. B 2011, 84, 235437. [CrossRef]

14. Li, G.X.; Li, T.; Liu, H.; Li, K.F.; Wang, S.M.; Zhu, S.N.; Cheah, K.W. Spectral analysis of enhanced third harmonic generation from plasmonic excitations. Appl. Phys. Lett. 2011, 98, 261909. [CrossRef]

15. West, P.; Ishii, S.; Naik, G.; Emani, N.K.; Shalaev, V.; Boltasseva, A. Searching for better plasmonic materials. Laser Photonics Rev. 2010, 4, 795-808. [CrossRef]

16. Naik, G.V.; Shalaev, V.M.; Boltasseva, A. Alternative Plasmonic Materials: Beyond Gold and Silver. Adv. Mater. 2013, 25, 3264-3294. [CrossRef]

17. Bouillard, J.-S.G.; Dickson, W.; O'Connor, D.P.; Wurtz, G.A.; Zayats, A.V. Low-Temperature Plasmonics of Metallic Nanostructures. Nano Lett. 2012, 12, 1561-1565. [CrossRef]

18. Lu, Y.-J.; Kim, J.; Chen, H.-Y.; Wu, C.; Dabidian, N.; Sanders, C.E.; Wang, C.-Y.; Lu, M.-Y.; Li, B.-H.; Qiu, X.; et al. Plasmonic Nanolaser Using Epitaxially Grown Silver Film. In Proceedings of the Conference on Lasers and Electro-Optics, San Jose, CA, USA, 6-11 May 2012. [CrossRef]

19. Naik, G.V.; Kim, J; Boltasseva, A. Oxides and nitrides as alternative plasmonic materials in the optical range [Invited]. Opt. Mater. Express 2011, 1, 1090-1099. [CrossRef]

20. Cortie, M.B.; McDonagh, A. Synthesis and Optical Properties of Hybrid and Alloy Plasmonic Nanoparticles. Chem. Rev. 2011, 111, 3713-3735. [CrossRef] [PubMed]

21. Gong, C.; Leite, M.S. Noble Metal Alloys for Plasmonics. ACS Photonics 2016, 3, 507-513. [CrossRef]

22. Gong, T.; Lyu, P.; Palm, K.J.; Memarzadeh, S.; Munday, J.N.; Leite, M.S. Emergent Opportunities with Metallic Alloys: From Material Design to Optical Devices. Adv. Opt. Mater. 2020, 8, 2001082. [CrossRef] 
23. Bobb, D.A.; Zhu, G.; Mayy, M.; Gavrilenko, A.V.; Mead, P.; Gavrilenko, V.I.; Noginov, M.A. Engineering of low-loss metal for nanoplasmonic and metamaterials applications. Appl. Phys. Lett. 2009, 95, 151102. [CrossRef]

24. Ching, S.Y. Plasmonic Properties of Silver-based Alloy Thin Films. Ph. D. Thesis, Hong Kong Baptist University, Hong Kong, China, November 2015.

25. Lai, S.L.; Chan, M.Y.; Fung, M.K.; Lee, C.; Hung, L.; Lee, S. Applications of Ytterbium in organic light-emitting devices as high performance and transparent electrodes. Chem. Phys. Lett. 2002, 366, 128-133. [CrossRef]

26. Xie, W.F.; Lau, K.C.; Lee, C.S.; Lee, S.T. Transparent organic light-emitting devices with LiF/Yb:Ag cathode. Thin Solid Films 2007, 515, 6975-6977. [CrossRef]

27. Li, G.; Li, J.; Tam, H.L.; Chan, C.T.; Cheah, K.W. Near field imaging with resonant cavity lens. Opt. Express 2010, 18, $2325-2331$. [CrossRef] [PubMed]

28. Li, G.; Li, J.; Tam, H.L.; Chan, C.T.; Cheah, K.W. Near Field Imaging from Multilayer Lens. J. Nanosci. Nanotechnol. 2011, 11, 10725-10728. [CrossRef] [PubMed]

29. Langford, J.I.; Wilson, A.J.C. Scherrer after sixty years: A survey and some new results in the determination of crystallite size. J. Appl. Crystallogr. 1978, 11, 102-113. [CrossRef]

30. Warren, B.E.; Muldawer, L. X-Ray Diffraction. Phys. Today 1970, 23, 53. [CrossRef]

31. Comsol Multiphysics; Comsol Inc.: Burlington, MA, USA, 2021. 